\title{
An extremal problem of regular simplices: the five-dimensional case
}

\author{
Ákos G. Horváth
}

\begin{abstract}
The new result of this paper is connected with the following problem: consider a supporting hyperplane of a regular simplex and its reflected image at this hyperplane. When will the volume of the convex hull of these two simplices be maximal? We prove that in the case when the dimension is less or equal to 4 , the maximal volume attained in the case when the hyperplane goes through on a vertex and is orthogonal to the height of the simplex at this vertex. More interesting that in the higher dimensional cases this position is not optimal. We also determine an optimal position of the hyperplane in the 5-dimensional case. This corrects an erroneous statement in my paper (Horváth in Beitr Geom Algebra 55(2):415-428, 2014).
\end{abstract}

Mathematics Subject Classification. 52A40, 52A38, 26B15, 52B11.

Keywords. Convex hull, isometry, reflection at a hyperplane, simplex, volume inequality.

\section{Introduction}

Assume that the intersecting simplices $S$ and $S_{H}$ are reflected copies of each other in the hyperplane $H$. Then $H$ intersects each of them in the same set. By the Main Lemma of paper [3] we have that the intersection of the simplices in an optimal case (when the volume of their common convex hull is maximal) is a common vertex. In the paper [3] we stated the following (see Theorem 3 in [3]): If $S$ is a regular simplex of dimension $n$, then $c\left(S, S^{H}\right):=$ $\frac{1}{\operatorname{Vol}_{n}(S)} \operatorname{Vol}\left(\operatorname{conv}\left(S, S^{H}\right)\right)=2 n$. The proof contained a wrong substitution implying partly-false result. We prove that this statement is true when $n \leq 4$ and it is false in the higher dimensional cases. Additionally, we solve the 5dimensional case and obtain the position of the simplex which gives maximal volume. Since this position cannot be generalized trivially for higher dimensions, we proposed the following open problem: 
Problem 1. Consider a supporting hyperplane $H$ of a regular $n$-simplex $S$ and denote by $S_{H}^{\prime}$ the orthogonal projection of $S$ to $H$. Determine the supporting hyperplane $H$ for the volume of $\operatorname{conv}\left\{S, S_{H}^{\prime}\right\}$ is maximal.

As we mentioned, Theorem 1 gives the answer in the cases when $n \leq 5$. We may mention here a very similar problem solved by P. Filliman. He investigated in $[1,2]$ the volume of the projection of a body using the exterior algebra method. He determined those supporting hyperplanes $H$ of the regular simplex $S$ for which the volume of $S_{H}^{\prime}$ is maximal or minimal, respectively. Similar interesting questions can be found in the paper [4] on the volume of the union of two convex bodies and also in the survey paper [5].

\section{Regular simplex}

First we give a sort of elementary computations regarding a $n$-dimensional regular simplex. The reader can be skipped this section if knows the mentioned results. ${ }^{1}$ Let us denote the vertices of the regular simplex of dimension $n$ by $\frac{1}{\sqrt{2}} e_{i}$, where $\left\{e_{0}, e_{1}, \ldots, e_{n}\right\}$ is an orthonormal basis of an $(n+1)$ dimensional Euclidean space. Let $s_{i}:=\frac{1}{\sqrt{2}}\left(e_{i}-e_{0}\right)$ for $i=0, \ldots, n$ the system of the vertices with respect to the $n$-dimensional hyperplane, $\bar{H}:=$ $\left\{\sum_{i=0}^{n} x_{i} e_{i}: \sum_{i=0}^{n} x_{i}=\frac{1}{\sqrt{2}}\right\}$. Then

$$
s=\frac{1}{\sqrt{2}} \sum_{i=1}^{n} s_{i}=\frac{1}{\sqrt{2}}\left(\begin{array}{c}
-n \\
1 \\
\vdots \\
1
\end{array}\right), \quad s_{2, k}=\frac{1}{\sqrt{2}} \sum_{i=1}^{k-1} s_{i}=\frac{1}{\sqrt{2}}\left(\begin{array}{c}
-(k-1) \\
1 \\
\vdots \\
1 \\
0 \\
\vdots \\
0
\end{array}\right)
$$

moreover we get the formulas on the vertices

$$
\begin{aligned}
s_{2, k}^{0} & =\frac{s_{2, k}}{\left\|s_{2, k}\right\|}, \quad c=\frac{1}{n+1} s, \\
\|s\| & =\sqrt{\frac{n(n+1)}{2}}, \quad\left\|s_{2, k}\right\|=\sqrt{\frac{(k-1) k}{2}}, \quad h=\frac{1}{n}\|s\|=\sqrt{\frac{n+1}{2 n}} \\
\|c\| & =\sqrt{\frac{n}{2(n+1)}},
\end{aligned}
$$

\footnotetext{
${ }^{1}$ Consider this part of the paper only hints. It is possible that the notation used here is used in later sections for different object.
} 
and finally the once on the normals

$$
u_{0}=\frac{s}{\|s\|}=\frac{1}{\sqrt{n(n+1)}}\left(\begin{array}{c}
-n \\
1 \\
\vdots \\
1
\end{array}\right), \quad u_{i}=\frac{c-s_{i}}{\left\|c-s_{i}\right\|}=\frac{1}{\sqrt{n(n+1)}}\left(\begin{array}{c}
1 \\
\vdots \\
1 \\
-n \\
1 \\
\vdots \\
1
\end{array}\right) .
$$

From the above results we can compute some further important formulas, we get

$$
\begin{aligned}
\left\langle s_{2, k}, u_{0}\right\rangle=\frac{(k-1)(n+1)}{\sqrt{2 n(n+1)}}, \quad\left\langle u_{i}, u_{j}\right\rangle=-\frac{1}{n}, & \left(\begin{array}{c}
-n+1 \\
1 \\
\vdots \\
1 \\
0 \\
1 \\
\vdots \\
1
\end{array}\right), \quad s-(n+1) s_{j}=\frac{1}{\sqrt{2}}\left(\begin{array}{c}
1 \\
\vdots \\
1 \\
-n \\
1 \\
\vdots \\
1
\end{array}\right)=\sqrt{\frac{n(n+1)}{2}} u_{j} .
\end{aligned}
$$

Assume that the $(n-1)$-dimensional flat $H$ of the hyperplane $\bar{H}$ through the vertex $s_{0}$ contains precisely the vertices $s_{0}, \ldots s_{r}$ and it is parallel to the affine hull of the remaining ones. Then the unit normal vector of $H$ directed towards to the interior of the half-space $H^{+}$which contains the simplex is

$$
\begin{aligned}
u & =\frac{1}{\sqrt{(n-r)(r+1)(n+1)}}\left(\sum_{i=0}^{r}-(n-r) e_{i}+\sum_{i=r+1}^{n+1}(r+1) e_{i}\right) \\
& =\frac{1}{\sqrt{(n-r)(r+1)(n+1)}}\left(\begin{array}{c}
-(n-r) \\
\vdots \\
-(n-r) \\
(r+1) \\
\vdots \\
r+1
\end{array}\right)
\end{aligned}
$$

and in this case we have

$$
\left\langle u, u_{0}\right\rangle=\sqrt{\frac{n-r}{r+1}} \frac{1}{\sqrt{n}} .
$$

Observe that $s_{0}$ always lies in $H$ while $s_{n}$ does not lie in it. Hence the inequality $0 \leq r \leq n-1$ holds. 


\section{The theorem}

In this section we prove the following theorem:

Theorem 1. If $S$ is the regular simplex of dimension $n \leq 4$, then

$$
c\left(S, S^{H}\right):=\frac{1}{\operatorname{Vol}_{n}(S)} \operatorname{Vol}\left(\operatorname{conv}\left(S, S^{H}\right)\right)=2 n,
$$

attained only in the case when $u=u_{0}=\frac{s}{\|s\|}$. For $n=5$ we have

$$
c\left(S, S^{H}\right)=10\left(\frac{1}{2}+\frac{\sqrt{77}}{10 \sqrt{3}}\right) \approx 10.06623>10=2 n .
$$

Proof. Without loss of generality we can assume that $\left\|s_{1}\right\|=\cdots=\left\|s_{n}\right\|=1$ as in the previous paragraph. We imagine that $H$ is horizontal and $H^{+}$is the upper half-space. Define the upper side of $S$ as the collection of those facets in which a ray orthogonal to $H$ and terminated in a far point of $H^{+}$is first intersecting $S$. The volume of the convex hull is twice the sum of the volumes of the prisms whose bases are on the orthogonal projection of the facets of the simplex of the upper side. Let $F_{i_{1}}, \ldots, F_{i_{k}}$ denotes the facets of the upper side, $F_{i_{1}}^{\prime}, \ldots, F_{i_{k}}^{\prime}$ their orthogonal projections on $H$, and $u_{i_{1}}, \ldots, u_{i_{k}}$ their respective unit normals, directed outwardly. Observe that in this case

$$
u_{i_{l}}= \begin{cases}\frac{(n+1) s_{i_{l}}-s}{\left\|(n+1) s_{i_{l}}-s\right\|} & \text { if } i_{l} \neq 0 \\ \frac{s}{\|s\|} & \text { if } i_{l}=0\end{cases}
$$

moreover

$$
\begin{aligned}
\left\|(n+1) s_{i_{l}}-s\right\| & =\left|\left\langle u_{i_{l}},(n+1) s_{i_{l}}-s\right\rangle\right|=\left|(n+1)\left\langle u_{i_{l}}, s_{i_{l}}\right\rangle-\left\langle u_{i_{l}}, s_{i_{l}}\right\rangle\right| \\
& =\sqrt{\frac{n(n+1)}{2}}\left\|s_{1}\right\| .
\end{aligned}
$$

We also introduced the notation $s=\sum_{i=0}^{n} s_{i}=\sum_{i=1}^{n} s_{i}$. Since $i_{1}=0$ corresponds to an upper facet, using Statement 2 in [3] we get

$$
\begin{aligned}
& \frac{\operatorname{Vol}\left(\operatorname{conv}\left(S, S^{H}\right)\right)}{\operatorname{Vol}_{n}(S)}=2 n \sum_{l=1}^{k} \frac{\left\langle u_{i_{l}}, u\right\rangle\left\langle u, s-s_{i_{l}}\right\rangle}{\left|\left\langle u_{i_{l}},(n+1) s_{i_{l}}-s\right\rangle\right|} \\
& =2 n\left(\left\langle u_{0}, u\right\rangle^{2}+\frac{2}{(n+1) n} \sum_{l=2}^{k}\left\langle-(n+1) s_{i_{l}}+s, u\right\rangle\left\langle u, s-s_{i_{l}}\right\rangle\right) \\
& =2 n\left(\left\langle u_{0}, u\right\rangle^{2}+\frac{2}{(n+1) n} \sum_{l=2}^{k}\left(-(n+1)\left\langle s_{i_{l}}, u\right\rangle+\langle s, u\rangle\right)\left(\langle u, s\rangle-\left\langle u, s_{i_{l}}\right\rangle\right)\right) \\
& =2 n\left(\left\langle u_{0}, u\right\rangle^{2}+\frac{2}{(n+1) n} \sum_{l=2}^{k}\left((n+1)\left\langle s_{i_{l}}, u\right\rangle^{2}-(n+2)\langle s, u\rangle\left\langle u, s_{i_{l}}\right\rangle+\langle u, s\rangle^{2}\right)\right) .
\end{aligned}
$$

If the only upper facet corresponds to the normal vector $u_{0}$, then only the first term occurs - meaning that $k=1$ - and the maximal value of the right hand side is less than or equal to $2 n$ with equality in the required case. 
Assume now that $k \geq 2$. By the regularity of the simplex we have that $s=$ $\sqrt{\frac{(n+1) n}{2}} u_{0}$, hence we get

$$
\begin{aligned}
& \frac{\operatorname{Vol}\left(\operatorname{conv}\left(S, S^{H}\right)\right)}{\operatorname{Vol}_{n}(S)}:=2 n f\left(\left\langle s_{2, k}^{0}, u\right\rangle,\left\langle u_{0}, u\right\rangle\right) \\
& =2 n\left(\left\langle u_{0}, u\right\rangle^{2}+\sum_{l=2}^{k}\left(\frac{2}{n}\left\langle s_{i_{l}}, u\right\rangle^{2}-\sqrt{\frac{2}{n(n+1)}}(n+2)\left\langle u_{0}, u\right\rangle\left\langle s_{i_{l}}, u\right\rangle+\left\langle u_{0}, u\right\rangle^{2}\right)\right) \\
& =2 n\left(\frac{2}{n} \sum_{l=2}^{k}\left\langle s_{i_{l}}, u\right\rangle^{2}-\sqrt{\frac{2}{n(n+1)}}(n+2)\left\langle u_{0}, u\right\rangle\left\langle\sum_{l=2}^{k} s_{i_{l}}, u\right\rangle+k\left\langle u_{0}, u\right\rangle^{2}\right)
\end{aligned}
$$

Set $s_{2, k}:=\sum_{l=2}^{k} s_{i_{l}}$ and $s_{2, k}^{0}:=\frac{s_{2, k}}{\left\|s_{2, k}\right\|}$, respectively.

Denote by $f$ the expression in the bracket then

$$
2 n f:=2 n\left(\frac{2}{n} \sum_{l=2}^{k}\left\langle s_{i_{l}}, u\right\rangle^{2}-\sqrt{\frac{(k-1) k}{n(n+1)}}(n+2)\left\langle u_{0}, u\right\rangle\left\langle s_{2, k}^{0}, u\right\rangle+k\left\langle u_{0}, u\right\rangle^{2}\right) .
$$

First we remark that the inequality $\frac{1}{n} \leq\left\langle u_{0}, u\right\rangle \leq 1$ is fulfilled. We can observe that if $F_{i_{l}}$ is an upper facet then

$$
\left\langle\sum_{i \neq i_{l}}\left(s_{i}-s_{i_{l}}\right), u\right\rangle \geq 0
$$

implying that

$$
\left\langle s-(n+1) s_{i_{l}}, u\right\rangle \geq 0 .
$$

From this we get a new connection between the parameters $\left\langle s_{i_{l}}, u\right\rangle$ and $\left\langle u_{0}, u\right\rangle$, namely

$$
\left\langle s_{i_{l}}, u\right\rangle \leq \frac{\|s\|}{(n+1)}\left\langle u_{0}, u\right\rangle=\sqrt{\frac{n}{2(n+1)}}\left\langle u_{0}, u\right\rangle .
$$

This implies that

$$
\frac{2}{n} \sum_{l=2}^{k}\left\langle s_{i_{l}}, u\right\rangle^{2} \leq \frac{(k-1)}{(n+1)}\left\langle u_{0}, u\right\rangle^{2} .
$$

On the other hand, if we write that

$$
\left\langle u_{0}, u\right\rangle:=\cos \alpha, \quad\left\langle s_{2, k}^{0}, u_{0}\right\rangle:=\cos \beta \text { and }\left\langle s_{2, k}^{0}, u\right\rangle:=\cos \gamma,
$$

then we get that $\gamma \leq \alpha+\beta$, and so $\cos \alpha \cos \beta-\sin \alpha \sin \beta \leq \cos \gamma$. But

$$
\cos \beta=\frac{(k-1)+\frac{1}{2}(k-1)(n-1)}{\sqrt{\frac{(k-1) k n(n+1)}{4}}}=\sqrt{1-\frac{n-k+1}{n k}} \text { and } \sin \beta=\sqrt{\frac{n-k+1}{n k}},
$$


hence we have a second inequality which is:

$$
\begin{aligned}
& \left\langle u_{0}, u\right\rangle \sqrt{\frac{(n+1)(k-1)}{n k}}-\sqrt{1-\left\langle u_{0}, u\right\rangle^{2}} \sqrt{\frac{n-k+1}{n k}} \leq\left\langle s_{2, k}^{0}, u\right\rangle \\
& =\sqrt{\frac{2}{k(k-1)} \sum_{l=2}^{k}\left\langle s_{i_{l}}, u\right\rangle .}
\end{aligned}
$$

Introduce the notation $x:=\left\langle u_{0}, u\right\rangle$. Now we get that

$$
\begin{aligned}
f \leq & \left(\left(\frac{k-1}{n+1}+k\right)-\frac{(k-1)(n+2)}{n}\right) x^{2} \\
& +\frac{(n+2)}{n} \sqrt{\frac{(k-1)(n-k+1)}{n+1}} x \sqrt{1-x^{2}} \\
=x & {\left[\left(1-(k-1)(n+2)\left(\frac{1}{n}-\frac{1}{n+1}\right)\right) x\right.} \\
& \left.+\frac{(n+2)}{n} \sqrt{\frac{(k-1)(n-k+1)}{n+1}} \sqrt{1-x^{2}}\right] \\
= & x\left[\left(1-\frac{(k-1)(n+2)}{n(n+1)}\right) x+\frac{(n+2)}{n} \sqrt{\frac{(k-1)(n-k+1)}{n+1}} \sqrt{1-x^{2}}\right] \\
= & x\left[A x+\sqrt{B} \sqrt{1-x^{2}}\right] .
\end{aligned}
$$

We have to prove that for all $x \in\left[\frac{1}{n}, 1\right], n \geq 3$ and $2 \leq k \leq n$ the inequality

$$
x\left[A x+\sqrt{B} \sqrt{1-x^{2}}\right]<1
$$

holds. We may assume that $x>0$. This inequality can be arranged to the form

$$
\frac{1}{x}-A x>\sqrt{B} \sqrt{1-x^{2}}
$$

Observe that the left hand side is greater than zero, because $0<A<1$ always hold. (This implies that $\frac{1}{x}-A x>\frac{1}{x}-x>0$.) Considering the square of the inequality we get the following one:

$$
\left(A^{2}+B\right) x^{4}-(2 A+B) x^{2}+1>0 .
$$

The possible roots of the quadric in the left hand side are

$$
\left(x^{2}\right)_{1,2}=\frac{(2 A+B) \pm \sqrt{B(4 A+B-4)}}{2\left(A^{2}+B\right)} .
$$

These are real numbers if and only if $4 A+B-4=B+4(A-1) \geq 0$. From this inequality we get

$$
0 \leq \frac{(n+2)^{2}}{n^{2}} \frac{(k-1)(n-k+1)}{n+1}-4 \frac{(k-1)(n+2)}{n(n+1)},
$$

equivalently

$$
4 \leq \frac{(n-k+1)(n+2)}{n}
$$


If $n=3$ then for $k=2,3$ the above inequality does not hold showing that the equation has no real solutions. This means that $f<1$ and the statement is true.

If $n=4$ than for $k=3,4$ the inequality (1) is false, too. However for $k=2$ it is hold, because $4<\frac{18}{4}$. In this case, $A=1-3 / 10=7 / 10, B=9 / 4 \cdot 3 / 5=27 / 20$ and the roots are

$$
x_{1,2}^{2}=\frac{7 / 5+27 / 20 \pm \sqrt{27 / 20(14 / 5+27 / 20-4)}}{2(49 / 100+27 / 20)}=\frac{11 / 4 \pm 9 / 20}{92 / 25},
$$

or simplifying there are

$$
x_{1}^{2}=20 / 23, \quad x_{2}^{2}=5 / 8,
$$

respectively. If the variable $x=\left\langle u_{0}, u\right\rangle$ lies between $\sqrt{5 / 8}$ and $\sqrt{20 / 23}$ then the examined function $f(x)$ can be greater or equal to 1 .

So we have to investigate the original formula in the following situation: Set $n=4, k=2$ and assume that $\sqrt{5 / 8} \leq\left\langle u_{0}, u\right\rangle \leq \sqrt{20 / 23}$. Since $s_{2, k}^{0}=s_{i, 2}$ we have the following inequalities between $\left\langle s_{i_{2}}, u\right\rangle$ and $\left\langle u_{0}, u\right\rangle$,

$$
\sqrt{\frac{2}{5}}\left\langle u_{0}, u\right\rangle \geq\left\langle s_{i_{2}}, u\right\rangle \geq\left\langle u_{0}, u\right\rangle \sqrt{\frac{5}{8}}-\sqrt{1-\left\langle u_{0}, u\right\rangle^{2}} \sqrt{\frac{3}{8}}
$$

The function

$$
f=\left(\frac{2}{n}\left\langle s_{i_{2}}, u\right\rangle^{2}-\sqrt{\frac{2}{n(n+1)}}(n+2)\left\langle u_{0}, u\right\rangle\left\langle s_{i_{2}}, u\right\rangle+k\left\langle u_{0}, u\right\rangle^{2}\right)
$$

is convex for a fixed value of $\left\langle u_{0}, u\right\rangle$ hence it can takes its maximal values at the ends of the corresponding interval. Hence we have to determine the values of $f$ using the conditions $\sqrt{\frac{2}{5}}\left\langle u_{0}, u\right\rangle=\left\langle s_{i_{2}}, u\right\rangle$ and $\left\langle s_{i_{2}}, u\right\rangle=\left\langle u_{0}, u\right\rangle \sqrt{\frac{5}{8}}-$ $\sqrt{1-\left\langle u_{0}, u\right\rangle^{2}} \sqrt{\frac{3}{8}}$, respectively. In the first case we get

$$
\left(\frac{1}{2}\left\langle s_{i_{2}}, u\right\rangle^{2}-3 \sqrt{\frac{2}{5}}\left\langle u_{0}, u\right\rangle\left\langle s_{i_{2}}, u\right\rangle+2\left\langle u_{0}, u\right\rangle^{2}\right)=\left(\frac{1}{5}-3 \frac{2}{5}+2\right)\left\langle u_{0}, u\right\rangle^{2} \leq 1,
$$

showing that $f(x) \leq 1$ as we stated. Secondly substitute the lower bound function into the expression of $f$. Using again the notation $x=\left\langle u_{0}, u\right\rangle$, we have to maximize the function

$$
g(x):=\frac{5}{8} x^{2}+\left(\sqrt{\frac{27}{20}}-\sqrt{\frac{15}{64}}\right) x \sqrt{1-x^{2}}+\frac{3}{16},
$$

on the interval $\sqrt{5 / 8} \leq x \leq \sqrt{20 / 23}$. It can be seen easily that on the interval $[1 / 2, \sqrt{20 / 23}]$ it is a concave function with an unique maximal value which is approximately $f(x) \approx 0.960977$ attends at the value $x=0.915944<\sqrt{20 / 23}$, proving our statement. 
Examine now the 5-dimensional case. The inequality (1) does not hold if $k>$ $6-20 / 7=4-6 / 7>3$. Thus we have to investigate two respective cases, when $k=2$ or $k=3$.

Let $k=3$. If we fix the value of $x:=\left\langle u_{0}, u\right\rangle$ the function

$$
\begin{aligned}
f= & \left(\frac{2}{5} \sum_{l=2}^{3}\left\langle s_{i_{l}}, u\right\rangle^{2}-\frac{7 \sqrt{15}}{15}\left\langle u_{0}, u\right\rangle\left\langle\sum_{l=2}^{3} s_{i_{l}}, u\right\rangle+3\left\langle u_{0}, u\right\rangle^{2}\right) \\
= & \left(\frac{2}{5}\left\langle s_{i_{2}}, u\right\rangle^{2}-\frac{7 \sqrt{15}}{15}\left\langle u_{0}, u\right\rangle\left\langle s_{i_{2}}, u\right\rangle+\frac{3}{2}\left\langle u_{0}, u\right\rangle^{2}\right) \\
& +\left(\frac{2}{5}\left\langle s_{i_{3}}, u\right\rangle^{2}-\frac{7 \sqrt{15}}{15}\left\langle u_{0}, u\right\rangle\left\langle s_{i_{3}}, u\right\rangle+\frac{3}{2}\left\langle u_{0}, u\right\rangle^{2}\right)
\end{aligned}
$$

is the sum of two convex functions defined on the same interval. The maximal value of the two terms separately can be achieved only at the ends of the interval. We have an upper bound for $f$ if we determine the maximal value of the two terms separately and we add them. The left end of the examined interval gave with the equality $\left\langle s_{i_{2}}, u\right\rangle=\left\langle u_{0}, u\right\rangle \sqrt{\frac{12}{5}}-\sqrt{1-\left\langle u_{0}, u\right\rangle^{2}} \sqrt{\frac{3}{5}}$ while the right end with the other one $\left\langle s_{i_{2}}, u\right\rangle=\sqrt{\frac{5}{12}}\left\langle u_{0}, u\right\rangle$. The sum of the two terms is less or equal to

$$
\begin{aligned}
2 \max & \left\{\left(\frac{2}{5}\left(\left\langle u_{0}, u\right\rangle \sqrt{\frac{12}{5}}-\sqrt{1-\left\langle u_{0}, u\right\rangle^{2}} \sqrt{\frac{3}{5}}\right)^{2}\right.\right. \\
& \left.-\frac{7 \sqrt{15}}{15}\left\langle u_{0}, u\right\rangle\left(\left\langle u_{0}, u\right\rangle \sqrt{\frac{12}{5}}-\sqrt{1-\left\langle u_{0}, u\right\rangle^{2}} \sqrt{\frac{3}{5}}\right)+\frac{3}{2}\left\langle u_{0}, u\right\rangle^{2}\right), \\
& \left.\left(\frac{1}{6}-\frac{7}{6}+\frac{3}{2}\right)\left\langle u_{0}, u\right\rangle^{2}\right\} \\
= & 2 \max \left\{-\frac{29}{50}\left\langle u_{0}, u\right\rangle^{2}+\frac{11}{25}\left\langle u_{0}, u\right\rangle \sqrt{1-\left\langle u_{0}, u\right\rangle^{2}}+\frac{6}{25}, \frac{1}{2}\left\langle u_{0}, u\right\rangle^{2}\right\} .
\end{aligned}
$$

The concave function

$$
h(x):=-\frac{29}{50} x^{2}+\frac{11}{25} x \sqrt{1-x^{2}}+\frac{6}{25}
$$

attends its maximal value 0.314005 on the interval $[0,1]$ at the point $x=$ 0.318833 showing that $f(x)<1$ in this case, too. ${ }^{2}$

The last case ${ }^{3}$ is when $n=5$ and $k=2$. The examined function $f$ is

$$
\frac{2}{5}\left\langle s_{i_{2}}, u\right\rangle^{2}-\frac{7 \sqrt{15}}{15}\left\langle u_{0}, u\right\rangle\left\langle s_{i, 2}, u\right\rangle+2\left\langle u_{0}, u\right\rangle^{2}
$$

\footnotetext{
${ }^{2}$ In this case we did not have to use the smaller domain, based on the sharper calculation of the values $A=8 / 15, B=196 / 75$ and $\left(x^{2}\right)_{1,2}=\frac{9(23 \pm 7 \sqrt{2})}{326}$.

${ }^{3}$ In this case $A=23 / 30, B=98 / 75$ and $\left(x^{2}\right)_{1,2}=\frac{23 / 15+98 / 75 \pm \sqrt{98 / 75(46 / 15+98 / 75-4)}}{2\left(23^{2} / 30^{2}+98 / 75\right)}$.
} 
and the conditions on the two variables are

$$
\left\langle u_{0}, u\right\rangle \sqrt{\frac{3}{5}}-\sqrt{1-\left\langle u_{0}, u\right\rangle^{2}} \sqrt{\frac{2}{5}} \leq\left\langle s_{i, 2}, u\right\rangle \leq \sqrt{\frac{5}{12}}\left\langle u_{0}, u\right\rangle .
$$

Hence we get again that

$$
f(x) \leq \max \left\{\frac{17}{25}\left\langle u_{0}, u\right\rangle^{2}+\frac{23 \sqrt{6}}{75}\left\langle u_{0}, u\right\rangle \sqrt{1-\left\langle u_{0}, u\right\rangle^{2}}+\frac{4}{25},\left\langle u_{0}, u\right\rangle^{2}\right\} .
$$

Consider the first argument of the right hand side and assume that $\left\langle s_{i, 2}, u\right\rangle=$ $\sin \varphi$ with a new variable $\varphi$. Then from the equality $\sin \varphi:=\left\langle u_{0}, u\right\rangle \sqrt{\frac{3}{5}}-$ $\sqrt{1-\left\langle u_{0}, u\right\rangle^{2}} \sqrt{\frac{2}{5}}$ we get that

$$
\left\langle u_{0}, u\right\rangle=\sqrt{\frac{3}{5}} \sin \varphi+\sqrt{\frac{2}{5}} \cos \varphi .
$$

The examined function now is

$$
\begin{aligned}
g(\varphi)= & \frac{17}{25}\left(\frac{3}{5} \sin ^{2} \varphi+\frac{2}{5} \cos ^{2} \varphi+2 \frac{\sqrt{6}}{5} \sin \varphi \cos \varphi\right) \\
& +\frac{23 \sqrt{6}}{75}\left(\sqrt{\frac{3}{5}} \sin \varphi+\sqrt{\frac{2}{5}} \cos \varphi\right)\left(\sqrt{\frac{3}{5}} \cos \varphi-\sqrt{\frac{2}{5}} \sin \varphi\right)+\frac{4}{25} \\
= & \left(\frac{51}{125}-6 \frac{23}{5^{3} 3}\right) \sin ^{2} \varphi+\left(\frac{34}{125}+6 \frac{23}{5^{3} 3}\right) \cos ^{2} \varphi \\
& +\left(2 \frac{17 \sqrt{6}}{125}+\frac{23 \sqrt{6}}{5^{3} 3}\right) \sin \varphi \cos \varphi+\frac{4}{25} \\
= & \frac{1}{5} \sin ^{2} \varphi+\frac{4}{5} \cos ^{2} \varphi+\frac{\sqrt{6}}{3} \sin \varphi \cos \varphi=\frac{3}{5} \cos ^{2} \varphi+\frac{\sqrt{6}}{3} \sin \varphi \cos \varphi+\frac{1}{5} .
\end{aligned}
$$

This function takes its maximal value at that point $\varphi_{\max }$ for which $\cos ^{2} \varphi_{\max }-$ $\sin ^{2} \varphi_{\max }=\sqrt{\frac{27}{77}}$ and $\sin \varphi_{\max } \cos \varphi_{\max }=\frac{1}{2} \sqrt{\frac{50}{77}}$, from which $\cos ^{2} \varphi_{\max }=$ $\frac{1}{2}\left(1+\sqrt{\frac{27}{77}}\right)$ and $\sin ^{2} \varphi_{\max }=\frac{1}{2}\left(1-\sqrt{\frac{27}{77}}\right)$. The maximal value is

$$
g\left(\varphi_{\max }\right)=\frac{1}{2}+\frac{3}{10} \sqrt{\frac{27}{77}}+\frac{10 \sqrt{3}}{6 \sqrt{77}}=\frac{1}{2}+\frac{\sqrt{77}}{10 \sqrt{3}} \approx 1.006623 .
$$

Since the corresponding values $\left\langle u_{0}, u\right\rangle$ and $\left\langle s_{i, 2}, u\right\rangle$ are allowed in our investigation this proves that obtained upper bound is attained. ${ }^{4}$ This proves the statement.

\footnotetext{
${ }^{4}$ Note that the equalities part of the inequalities connect to real positions of the regular simplex, hence in these cases we have such placements of the regular simplex which give the corresponding values. However we didn't investigate the realizability of the another pairs of the parameters or the problem that the maximal volume can be or cannot be realized in another way.
} 
Remark. An optimal position of the regular simplex can be written geometrically in the five-dimensional case, too. We start with the position of the simplex, when the edge joining the vertices $s_{0}$ and $s_{1}$ lies in $H$ and the affine hull $G$ of the remaining vertices is parallel to $H$. The maximal volume can be computed if we rotate this simplex around the orthogonal direct component $s_{1}^{\perp}$ of the line of $s_{1}$ with respect to $H$ by the angle $\varphi_{\max }$. Before the rotation we have that

$$
\begin{aligned}
& s_{0}=\frac{1}{\sqrt{2}}\left(\begin{array}{l}
1 \\
0 \\
0 \\
0 \\
0 \\
0
\end{array}\right), \quad s_{1}=\frac{1}{\sqrt{2}}\left(\begin{array}{c}
-1 \\
1 \\
0 \\
0 \\
0 \\
0
\end{array}\right), \quad s_{2}=\frac{1}{\sqrt{2}}\left(\begin{array}{c}
-1 \\
0 \\
1 \\
0 \\
0 \\
0
\end{array}\right), \quad s_{3}=\frac{1}{\sqrt{2}}\left(\begin{array}{c}
-1 \\
0 \\
0 \\
1 \\
0 \\
0
\end{array}\right), \\
& s_{4}=\frac{1}{\sqrt{2}}\left(\begin{array}{c}
-1 \\
0 \\
0 \\
0 \\
1 \\
0
\end{array}\right), \quad s_{5}=\frac{1}{\sqrt{2}}\left(\begin{array}{c}
-1 \\
0 \\
0 \\
0 \\
0 \\
1
\end{array}\right), \quad u=\frac{1}{2 \sqrt{3}}\left(\begin{array}{c}
-2 \\
-2 \\
1 \\
1 \\
1 \\
1
\end{array}\right), \quad u_{0}=\frac{1}{\sqrt{30}}\left(\begin{array}{c}
-5 \\
1 \\
1 \\
1 \\
1 \\
1
\end{array}\right) .
\end{aligned}
$$

Since $s_{1}$ is in the 2-plane which is spanned by $u$ and $u_{0}$ we have the equality $u_{0}=\frac{\sqrt{15}}{5} s_{1}+\frac{\sqrt{10}}{5} u$. Our rotation restricted to the 4 -subspace $\left\langle\left\{s_{1}^{\perp}, \sum_{i=1}^{6} e_{i}\right\}\right\rangle$ is the identity and on the 2 -subspace is generated by $s_{1}$ and $u$ it acts as a standard rotation. We consider the new orthonormal basis $\left\{\frac{1}{\sqrt{6}} \sum_{i=1}^{6} e_{i}, f_{1}, f_{2}, f_{3}, s_{1}, u\right\}$ where $\left\{f_{1}, f_{2}, f_{3}\right\}$ is an orthonormal basis of $s_{1}^{\perp}$. Clearly, $\left\langle\left\{s_{5}-s_{2}, s_{5}-s_{3}, s_{5}-\right.\right.$ $\left.\left.s_{4}\right\}\right\rangle=\left\langle\left\{f_{1}, f_{2}, f_{3}\right\}\right\rangle$ hence we can choose $f_{1}$ to $s_{5}-s_{2}, f_{2}$ to $\left(s_{5}-s_{3}\right)-\left(s_{5}-\right.$ $\left.s_{4}\right)=s_{4}-s_{3}$ and $f_{3}$ to $\frac{1}{\sqrt{2}}\left(\left(-\left(s_{5}-s_{2}\right)+\left(s_{5}-s_{3}\right)+\left(s_{5}-s_{4}\right)\right)\right.$, respectively. The orthogonal matrix of the basis change is

$$
B=\left(\begin{array}{cccccc}
\frac{1}{\sqrt{6}} & 0 & 0 & 0 & -\frac{1}{\sqrt{2}} & -\frac{1}{\sqrt{3}} \\
\frac{1}{\sqrt{6}} & 0 & 0 & 0 & \frac{1}{\sqrt{2}} & -\frac{1}{\sqrt{3}} \\
\frac{1}{\sqrt{6}} & -\frac{1}{\sqrt{2}} & 0 & \frac{1}{2} & 0 & \frac{1}{2 \sqrt{3}} \\
\frac{1}{\sqrt{6}} & 0 & -\frac{1}{\sqrt{2}} & -\frac{1}{2} & 0 & \frac{1}{2 \sqrt{3}} \\
\frac{1}{\sqrt{6}} & 0 & \frac{1}{\sqrt{2}} & -\frac{1}{2} & 0 & \frac{1}{2 \sqrt{3}} \\
\frac{1}{\sqrt{6}} & \frac{1}{\sqrt{2}} & 0 & \frac{1}{2} & 0 & \frac{1}{2 \sqrt{3}}
\end{array}\right) .
$$

Since $B^{-1}=B^{T}$ we can get easily the new coordinates of the vertices. The new coordinates of the vertex $\frac{1}{\sqrt{2}} e_{i}$ are the elements of the $i$-th row of $B$ multiply by $1 / \sqrt{2}$, respectively. The translation of the origin to the first vertex $\frac{1}{\sqrt{2}} e_{1}$ of the simplex is equivalent to the subtraction of the first column of $\frac{1}{\sqrt{2}} B^{T}$ from 
the column vector of it, thus the new coordinates of the examined vectors are

$$
\begin{aligned}
& s_{0}=\left(\begin{array}{l}
0 \\
0 \\
0 \\
0 \\
0 \\
0
\end{array}\right), \quad s_{1}=\left(\begin{array}{l}
0 \\
0 \\
0 \\
0 \\
1 \\
0
\end{array}\right), \quad s_{2}=\left(\begin{array}{c}
0 \\
-\frac{1}{2} \\
0 \\
\frac{1}{\sqrt{8}} \\
\frac{1}{2} \\
\sqrt{\frac{3}{8}}
\end{array}\right), \quad s_{3}=\left(\begin{array}{c}
0 \\
0 \\
-\frac{1}{2} \\
-\frac{1}{\sqrt{8}} \\
\frac{1}{2} \\
\sqrt{\frac{3}{8}}
\end{array}\right), \\
& s_{4}=\left(\begin{array}{c}
0 \\
0 \\
-\frac{1}{\sqrt{8}} \\
\frac{1}{2} \\
\sqrt{\frac{3}{8}}
\end{array}\right), \quad s_{5}=\left(\begin{array}{c}
0 \\
\frac{1}{2} \\
0 \\
\frac{1}{\sqrt{8}} \\
\frac{1}{2} \\
\sqrt{\frac{3}{8}}
\end{array}\right), \quad u_{0}=\left(\begin{array}{c}
0 \\
0 \\
0 \\
0 \\
\frac{\sqrt{15}}{5} \\
\frac{\sqrt{10}}{5}
\end{array}\right), \quad u=\left(\begin{array}{l}
0 \\
0 \\
0 \\
0 \\
1
\end{array}\right) .
\end{aligned}
$$

The matrix of the rotation and the respective rotated vertices are

$$
\begin{aligned}
& \left(\begin{array}{cccccc}
1 & 0 & 0 & 0 & 0 & 0 \\
0 & 1 & 0 & 0 & 0 & 0 \\
0 & 0 & 1 & 0 & 0 & 0 \\
0 & 0 & 0 & 1 & 0 & 0 \\
0 & 0 & 0 & 0 & \cos \varphi & -\sin \varphi \\
0 & 0 & 0 & 0 & \sin \varphi & \cos \varphi
\end{array}\right) \\
& s_{0}(\varphi)=\left(\begin{array}{l}
0 \\
0 \\
0 \\
0 \\
0 \\
0
\end{array}\right), \quad s_{1}(\varphi)=\left(\begin{array}{c}
0 \\
0 \\
0 \\
0 \\
\cos \varphi \\
\sin \varphi
\end{array}\right), \quad s_{2}(\varphi)=\left(\begin{array}{c}
0 \\
-\frac{1}{2} \\
0 \\
\frac{1}{\sqrt{8}} \\
\frac{1}{2} \cos \varphi-\sqrt{\frac{3}{8}} \sin \varphi \\
\frac{1}{2} \sin \varphi+\sqrt{\frac{3}{8}} \cos \varphi
\end{array}\right) \text {, } \\
& s_{3}(\varphi)=\left(\begin{array}{c}
0 \\
0 \\
-\frac{1}{2} \\
-\frac{1}{\sqrt{8}} \\
\frac{1}{2} \cos \varphi-\sqrt{\frac{3}{8}} \sin \varphi \\
\frac{1}{2} \sin \varphi+\sqrt{\frac{3}{8}} \cos \varphi
\end{array}\right), \quad s_{4}(\varphi)=\left(\begin{array}{c}
0 \\
0 \\
\frac{1}{2} \\
-\frac{1}{\sqrt{8}} \\
\frac{1}{2} \cos \varphi-\sqrt{\frac{3}{8}} \sin \varphi \\
\frac{1}{2} \sin \varphi+\sqrt{\frac{3}{8}} \cos \varphi
\end{array}\right) \text {, } \\
& s_{5}(\varphi)=\left(\begin{array}{c}
0 \\
\frac{1}{2} \\
0 \\
\frac{1}{\sqrt{8}} \\
\frac{1}{2} \cos \varphi-\sqrt{\frac{3}{8}} \sin \varphi \\
\frac{1}{2} \sin \varphi+\sqrt{\frac{3}{8}} \cos \varphi
\end{array}\right)
\end{aligned}
$$


Since we also have

$$
u_{0}(\varphi)=\left(\begin{array}{c}
0 \\
0 \\
0 \\
0 \\
\frac{\sqrt{15}}{5} \cos \varphi-\frac{\sqrt{10}}{5} \sin \varphi \\
\frac{\sqrt{15}}{5} \sin \varphi+\frac{\sqrt{10}}{5} \cos \varphi
\end{array}\right) \text { and } u=\left(\begin{array}{l}
0 \\
0 \\
0 \\
0 \\
0 \\
1
\end{array}\right)
$$

we get the calculation of the previous paragraph showing that the maximal volume attained at $\cos \varphi_{\max }=\sqrt{\frac{1}{2}\left(1+\sqrt{\frac{27}{77}}\right)}$. Using the notation where $a:=$ $\left(1+\sqrt{\frac{27}{77}}\right)$ and $b:=\left(1-\sqrt{\frac{27}{77}}\right)$, we get that the eleven vertices of the optimal polyhedron are

$$
\begin{aligned}
& \left.\left(\begin{array}{l}
0 \\
0 \\
0 \\
0 \\
0 \\
0
\end{array}\right), \quad\left(\begin{array}{c}
0 \\
0 \\
0 \\
0 \\
\sqrt{\frac{1}{2} a} \\
\pm \sqrt{\frac{1}{2} b}
\end{array}\right), \quad\left(\begin{array}{c}
0 \\
-\frac{1}{2} \\
0 \\
\frac{1}{\sqrt{8}} \\
\sqrt{\frac{1}{8} a}-\sqrt{\frac{3}{16} b} \\
\pm\left(\sqrt{\frac{3}{16} a}+\sqrt{\frac{1}{8} b}\right)
\end{array}\right), \quad \begin{array}{c}
0 \\
0 \\
-\frac{1}{2} \\
-\frac{1}{\sqrt{8}} \\
\sqrt{\frac{1}{8} a}-\sqrt{\frac{3}{16} b} \\
\pm\left(\sqrt{\frac{3}{16} a}+\sqrt{\frac{1}{8} b}\right)
\end{array}\right), \\
& \left(\begin{array}{c}
0 \\
0 \\
\frac{1}{2} \\
-\frac{1}{\sqrt{8}} \\
\pm \sqrt{\frac{1}{8} a}-\sqrt{\frac{3}{16} b} \\
\left. \pm \sqrt{\frac{3}{16} a}+\sqrt{\frac{1}{8} b}\right)
\end{array}\right), \quad\left(\begin{array}{c}
0 \\
\frac{1}{2} \\
0 \\
\frac{1}{\sqrt{8}} \\
\sqrt{\frac{1}{8} a}-\sqrt{\frac{3}{16} b} \\
\pm\left(\sqrt{\frac{3}{16} a}+\sqrt{\frac{1}{8} b}\right)
\end{array}\right)
\end{aligned}
$$

respectively. The normals of the upper facets are

$$
u_{0}\left(\varphi_{\max }\right)=\left(\begin{array}{c}
0 \\
0 \\
0 \\
0 \\
\sqrt{\frac{3}{10} a}-\sqrt{\frac{2}{10} b} \\
\sqrt{\frac{2}{10} a}+\sqrt{\frac{3}{10} b}
\end{array}\right) \text { and } u_{1}\left(\varphi_{\max }\right)=\left(\begin{array}{c}
0 \\
0 \\
0 \\
0 \\
-\sqrt{\frac{3}{10} a}-\sqrt{\frac{2}{10} b} \\
\sqrt{\frac{2}{10} a}-\sqrt{\frac{3}{10} b}
\end{array}\right) .
$$

We can imagine the corresponding body as pairwise non-overlapping union of two pyramids and a simplex. The common base of the pyramids is a fourdimensional prism defined by the convex hull of the vertices $s_{2}\left(\varphi_{\max }\right), s_{3}\left(\varphi_{\max }\right)$, $s_{4}\left(\varphi_{\max }\right), s_{5}\left(\varphi_{\max }\right)$ and its reflected images $s_{2}^{\prime}\left(\varphi_{\max }\right), s_{3}^{\prime}\left(\varphi_{\max }\right), s_{4}^{\prime}\left(\varphi_{\max }\right)$, $s_{5}^{\prime}\left(\varphi_{\max }\right)$ with 4 -dimensional volume

$$
\left(\frac{1}{6} \cdot 1 \cdot 1 \cdot 1 \cdot \frac{1}{\sqrt{2}}\right) 2\left(\sqrt{\frac{3}{16} a}+\sqrt{\frac{1}{8} b}\right)=\frac{1}{3}\left(\sqrt{\frac{3}{32} a}+\sqrt{\frac{1}{16} b}\right) .
$$


The apex of the first pyramid is $s_{0}$, while of the second one is $s_{1}\left(\varphi_{\max }\right)$. From this we get that the volume of the union is

$$
\begin{gathered}
\frac{1}{5} \sqrt{\frac{1}{2} a} \cdot \frac{1}{3}\left(\sqrt{\frac{3}{32} a}+\sqrt{\frac{1}{16} b}\right)=\frac{1}{3 \cdot 5}\left(\sqrt{\frac{3}{64} a+\sqrt{\frac{1}{32} a b}}\right) \\
=\frac{1}{3 \cdot 5}\left(\frac{\sqrt{3}}{8}\left(1+\sqrt{\frac{27}{77}}\right)+\sqrt{\frac{1}{32} \frac{50}{77}}\right)=\frac{1}{5 !}\left(\sqrt{3}+\frac{19}{\sqrt{77}}\right) .
\end{gathered}
$$

The last part is that simplex which defined by the vertices $\pm s_{1}\left(\varphi_{\max }\right), s_{2}^{\prime}\left(\varphi_{\max }\right)$, $s_{3}^{\prime}\left(\varphi_{\max }\right), s_{4}^{\prime}\left(\varphi_{\max }\right), s_{5}^{\prime}\left(\varphi_{\max }\right)$. Its volume is $\frac{1}{4 \cdot 5 !}\left(\sqrt{3}+\frac{1}{\sqrt{77}}\right)$ giving the volume of the body:

$$
v=\frac{1}{4 \cdot 5 !}(5 \sqrt{3}+\sqrt{77}) .
$$

Since the volume of the regular simplex of dimension 5 with edge length 1 is $\frac{\sqrt{3}}{4 \cdot 5 !}$ we get immediately again the ratio $10\left(\frac{1}{2}+\frac{\sqrt{77}}{10 \sqrt{3}}\right)$ of the previous paragraph.

\section{Acknowledgements}

Open access funding provided by Budapest University of Technology and Economics (BME). I am thankful to my colleague Zsolt Lángi who found the mistake in the proof of Theorem 3 of paper [3] and inspired me to write the present ones. I also thank for the helpful suggestions of Hans Havlicek and the unknown referee.

Open Access. This article is distributed under the terms of the Creative Commons Attribution 4.0 International License (http://creativecommons.org/licenses/ by/4.0/), which permits unrestricted use, distribution, and reproduction in any medium, provided you give appropriate credit to the original author(s) and the source, provide a link to the Creative Commons license, and indicate if changes were made.

Publisher's Note Springer Nature remains neutral with regard to jurisdictional claims in published maps and institutional affiliations.

\section{References}

[1] Filliman, P.: Exterior algebra and projections of polytopes. Discrete Comput. Geom. 5, 305-322 (1990)

[2] Filliman, P.: The extreme projections of the regular simplex. Trans. Am. Math. Soc. 317(2), 611-629 (1990)

[3] Horváth, Á.G.: On an extremal problem connected with simplices. Beitr. Geom. Algebra 55(2), 415-428 (2014) 
[4] Horváth, Á.G., Lángi, Z.: On the volume of the convex hull of two convex bodies. Monatsh. Math. 174(2), 219-229 (2014)

[5] Horváth, Á.G.: Volume of convex hull of two bodies and related problems. In: Conder, M., Deza, A., Weiss, A.I. (eds.) Discrete Geometry and Symmetry. Springer Proceedings in Mathematics and Statistics, vol. 234. Springer, Cham (2018). ISBN: 978-3-319-78433-5

\author{
Ákos G. Horváth \\ Department of Geometry, Mathematical Institute \\ Budapest University of Technology and Economics \\ Budapest 1521 \\ Hungary \\ e-mail: ghorvath@math.bme.hu
}

Received: November 30, 2018.

Revised: March 13, 2019. 